\title{
TWIN CONVERGENCE REGIONS FOR CONTINUED FRACTIONS
}

V. F. COWLING, WALTER LEIGHTON, AND W. J. THRON

1. Introduction. Some years ago it was proved $[2]^{1}$ that the continued fraction

$$
1+\frac{a_{1}}{1}+\frac{a_{2}}{1}+\cdots
$$

converges if the complex numbers $a_{n}$ satisfy the following conditions:

$$
\left|a_{2 n+1}\right| \leqq 1 / 4, \quad\left|a_{2 n}\right| \geqq 25 / 4 \quad(n=1,2, \cdots) .
$$

Later one of the present authors proved [3] that (1.1) converges if

$$
\begin{aligned}
\left|1+a_{2}\right| & \geqq 1+\left|a_{1}\right|, \\
\left|1+a_{n}+a_{n+1}\right| & \geqq 1+\left|a_{n-1} a_{n}\right| \quad(n=2,3, \cdots) .
\end{aligned}
$$

One of the immediate consequences of this theorem is that if

$$
\begin{aligned}
& \left|1+a_{2}\right|>1, \quad\left|a_{2}\right| \geqq(2+m) /(1-m), \\
& \left|a_{2 n+1}\right| \leqq m<1, \quad\left|a_{2 n+2}\right| \geqq 2+m+m\left|a_{2 n}\right| \\
& (n=1,2,3, \cdots) \text {, }
\end{aligned}
$$

then (1.1) converges.

Recently Thron [6] has shown that if

$$
\left|a_{2 n+1}\right| \leqq k^{2}<1, \quad\left|a_{2 n}\right| \geqq(1+k)^{2}+6 \quad(e>0),
$$

the continued fraction (1.1) converges. For $k^{2}<1$ this result can be shown to be a "best" result except possibly for the presence of the quantity $e$.

The present paper is concerned with establishing convergence criteria of this general type. The principal result is given in Theorem 3.1. The method to be used is the following. Denote the $n$th approximant of (1.1) by $A_{n} / B_{n}$. Conditions on the numbers $a_{n}$ are determined which imply that the approximants lie in a given region $V$ of the complex plane. A continued fraction the elements of which are functions of the complex variable $z$ and which reduces to (1.1) for $z=1$ is then introduced. When the given conditions on the numbers $a_{n}$ are satisfied the approximants of this continued fraction are shown to

Presented to the Society, September 13,1943, under the title On convergence regions for continued fractions; received by the editors June 1, 1943.

${ }^{1}$ Numbers in brackets refer to the Bibliography at the end of the paper. 
form a normal family for $z$ in a region $D$ of the complex plane which includes the point $z=1$. From the known convergence of this family in a sub-region of $D$ the (uniform) convergence of the family in every closed region interior to $D$ may be inferred. The convergence of (1.1) is then trivial.

2. Two lemmas. It will be convenient to refer to an open connected set as an open region and to use the term region to mean an open region plus part or all its boundary. If $S$ is a set of complex numbers and $a$ is a fixed complex number, we shall designate by $a+S$ and by $S+a$ the set obtained by adding to each element of $S$ the number $a$. Geometrically it is clear that $a+S$ is a translation of the set $S$ by means of the vector $a$. Similarly $a S$ will denote the set obtained from $S$ by multiplying each element of $S$ by the number $a$. Geometrically this constitutes a stretching of the set $S$ followed by a rotation of the resulting set through an angle of $\arg a$. By the notation $D[a S]$ is meant the point set intersection of all sets $a S$ as $a$ assumes all values in a given $\operatorname{set} A$.

The following lemma is an extension of a result due to Scott and Wall [4].

Lemma 2.1. Let $D_{1}, D_{2}, V_{1}, V_{2}$ be any four sets of points in the complex plane which have the following properties:

(a) $1+D_{1} \subset V_{1}$,

(b) $1+D_{2} \subset V_{2}$,

(c) $1+a / v \in V_{1}$, if $a \in D_{1}$, and $v \in V_{2}$,

(d) $1+a / v \in V_{2}$, if $a \in D_{2}$, and $v \in V_{1}$.

If the numbers $a_{2 n-1} \in D_{1}$ and $a_{2 n} \in D_{2}(n=1,2, \cdots)$, the values of the approximants of the continued fraction (1.1) are numbers which are contained in $V_{1}$, and the values of the approximants of

$$
1+\frac{a_{2}}{1}+\frac{a_{3}}{1}+\cdots
$$

belong to $V_{2}$.

The proof of this lemma is by an easy induction.

We proceed with the proof of the following result which is a consequence of Lemma 2.1.

LEMMA 2.2. Let $V_{1}$ and $V_{2}$ be any two sets of numbers in the complex plane such that the sets

$$
\begin{aligned}
& E_{1}=D\left[v_{2}\left(V_{1}-1\right)\right], \\
& E_{2}=D\left[v_{1}\left(V_{2}-1\right)\right]
\end{aligned}
$$


have the property that

$$
1+E_{1} \subset V_{1}, \quad 1+E_{2} \subset V_{2} \text {. }
$$

If the numbers $a_{2 n-1} \in E_{1}$ and $a_{2 n} \in E_{2}(n=1,2, \cdots)$, the values of the approximants of (1.1) belong to $V_{1}$ and the values of the approximants of (2.1) belong to $V_{2}$.

To prove this lemma it will be sufficient to show that conditions (c) and (d) of Lemma 2.1 are satisfied when we have identified the sets $D_{i}$ of that lemma with the present sets $E_{i}$, for $i=1$, 2, respectively. We shall prove that condition (c) holds. The proof for (d) will then follow mutatis mutandis. To that end let $a$ be any point belonging to $E_{1}$ and $v_{2}$ be any point belonging to $V_{2}$ and observe that the definition of $E_{1}$ insures the existence of a point $v^{\prime}{ }_{1} \in V_{1}$ such that $a=v_{2}\left(v_{1}^{\prime}-1\right)$. It follows that $1+a / v_{2}=v_{1}^{\prime} \in V_{1}$.

3. The principal theorem. We proceed with the proof of the following result.

THEOREM 3.1. Let $k$ be any real number greater than 1 and let $\epsilon$ and $\epsilon_{1}$ be positive numbers less than $k$. If the elements $a_{n}=r_{n} e^{i \theta n}$ are such that

$$
r_{2 n} \geqq 2\left[k+\epsilon_{1}-\cos \theta_{2 n}\right], \quad r_{2 n-1} \leqq k-\epsilon \quad(n=1,2, \cdots),
$$

the continued fraction (1.1) converges.

The first step in the demonstration of this theorem is the proof of the following lemma.

LEMMA 3.1. Let $k$ be any real number greater than 1 . If the elements $a_{n}=r_{n} e^{i \theta n}$ are such that

$$
r_{2 n-1} \leqq k, \quad r_{2 n} \geqq 2\left(k-\cos \theta_{2 n}\right) \quad\left(0 \leqq \theta_{2 n} \leqq 2 \pi ; n=1,2, \cdots\right),
$$

the values of the approximants of (1.1) belong to $V_{1}$ and the values of the approximants of (2.1) belong to $V_{2}$.

Let the sets $V_{1}$ and $V_{2}$ of Lemma 2.2 be respectively the sets $|z-1| \leqq k$ and $|z| \geqq 1$. It will follow that (i) the set $E_{1}=D\left[v_{2}\left(V_{1}-1\right)\right]$ is the set $|z| \leqq k$ and that (ii) $E_{2}=D\left[v_{1}\left(V_{2}-1\right)\right]$ is the set of points $z=r e^{i \theta}$ for which $r \geqq 2(k-\cos \theta)$.

To prove (i) observe that $V_{1}-1$ is the set of points $z$ for which $|z| \leqq k$ and that the set $v_{2}^{\prime}\left(V_{1}-1\right) \subset v_{2}\left(V_{1}-1\right)$ whenever $v_{2}$ and $v_{2}^{\prime}$ are elements of $V_{2}$ such that arg $v_{2}^{\prime}=\arg v_{2}$ and $\left|v_{2}^{\prime}\right|<\left|v_{2}\right|$. For in these circumstances the set $v_{2}\left(V_{1}-1\right)$ is the circular region defined by $|z| \leqq k\left|v_{2}\right|$ while the set $v_{2}^{\prime}\left(V_{1}-1\right)$ is the region $|z| \leqq k\left|v_{2}^{\prime}\right|=k$. Thus 
the set $E_{1}$ is the set $|z| \leqq k$. We note that

$$
1+E_{1} \subset V_{1} \text {. }
$$

The proof of (ii) is somewhat more complicated. We observe that $V_{2}-1$ is the set of complex numbers $z$ for which $|z+1| \geqq 1$. The set $0(V-1)$ is not well-defined. From continuity considerations we shall define it to be the set exterior to the null circle $|z|=0$. Consider then the set of products $v_{1}\left(V_{2}-1\right)$ where $v_{1} \in V_{1}$ and $v_{1} \neq 0$. If $v_{1}$ and $v_{1}^{\prime}$ are any two such elements of $V_{1}$ having the properties arg $v_{1}=\arg v_{1}^{\prime}$ and $\left|v_{1}^{\prime}\right|>\left|v_{1}\right|$, then $v_{1}^{\prime}\left(V_{2}-1\right) \subset v_{1}\left(V_{2}-1\right)$. This is true since multiplication of $V_{2}-1$ by $v_{1}^{\prime}$ subjects the set to the same rotation as multiplication by $v_{1}$. Since however $\left|v_{1}^{\prime}\right|>\left|v_{1}\right|$, the "exterior" set which is $v_{1}\left(V_{2}-1\right)$ contains the "exterior" set $v_{1}^{\prime}\left(V_{2}-1\right)$. Thus $D\left[v_{1}^{\prime}\left(V_{2}-1\right)\right]$ is the set $D\left[v_{1}\left(V_{2}-1\right)\right]=E_{2}$ for $v_{1}^{\prime}$ on the boundary of $V_{1}$. We can now prove that $E_{2}$ is the set of numbers $z=r e^{i \theta}$ for which

$$
r \geqq 2(k-\cos \theta) \text {. }
$$

To determine the boundary of $E_{2}$ it is clearly sufficient to consider only those products $v_{1}^{\prime}\left(v_{2}^{\prime}-1\right)$ where $v_{1}^{\prime}$ and $v_{2}^{\prime}$ range over the boundaries of $V_{1}$ and $V_{2}$ respectively. To that end let $r(\alpha) e^{i \alpha}$ and $\rho(\beta) e^{i \beta}$ be arbitrary points of the boundaries of $V_{1}$ and $V_{2}-1$, respectively. It is clear that

$$
\begin{array}{lr}
r(\alpha)=\cos \alpha+\left(k^{2}-\sin ^{2} \alpha\right)^{1 / 2} & (0 \leqq \alpha \leqq 2 \pi), \\
\rho(\beta)=-2 \cos \beta & (\pi / 2 \leqq \beta \leqq 3 \pi / 2) .
\end{array}
$$

We shall show first that

$$
r(\alpha) \rho(\beta) \leqq 2[k-\cos (\alpha+\beta)] \quad(\text { all } \alpha, \beta) .
$$

It will follow then that the boundary of $E_{2}$ is not exterior to the limaçon $r=2(k-\cos \theta)$. The proof of (3.2) is easy. It is clearly suffcient to prove that

$$
-\cos \beta\left(k^{2}-\sin ^{2} \alpha\right)^{1 / 2} \leqq k+\sin \alpha \sin \beta .
$$

Since $\cos \beta<0$ and $k>1$, it is then sufficient to prove that

$$
\cos ^{2} \beta\left(k^{2}-\sin ^{2} \alpha\right) \leqq(k+\sin \alpha \sin \beta)^{2} .
$$

But this is equivalent to

$$
0 \leqq(k \sin \beta+\sin \alpha)^{2} .
$$

Thus (3.2) is true. However, we observe that inequalities (3.2) and (3.3) are equivalent. With this in mind we can establish that corresponding to each angle $\alpha+\beta=\theta$ there exists an essentially unique 
choice of $\alpha$ and of $\beta$ such that the equality sign in (3.2) and in (3.3) holds. It will follow that the limaçon $r=2(k-\cos \theta)$ is the boundary of $E_{2}$. The angles $\alpha$ and $\beta$ referred to are determined by the following conditions:

$$
\begin{array}{ll}
\sin \alpha=-k \sin \theta / \Delta, & \cos \alpha=(1-k \cos \theta) / \Delta, \\
\sin \beta=\sin \theta / \Delta, & \cos \beta=(\cos \theta-k) / \Delta,
\end{array}
$$

where $\Delta=\left(1-2 k \cos \theta+k^{2}\right)^{1 / 2}$.

It is clear that these equations determine $\alpha$ and $\beta$ to within an additive integral multiple of $2 \pi$. It is easy to see that (3.3) and hence (3.2) reduces to an equality for this choice of $\alpha$ and $\beta$. It is not diffcult to prove that there is no other choice of $\alpha$ and $\beta$ having the desired properties. However, since the uniqueness is irrelevant to our present discussion, its proof is omitted.

The proof of the lemma is completed by noting that

$$
1+E_{2} \subset V_{2}
$$

and recalling (3.1).

To complete the proof of Theorem 3.1 we introduce the continued fraction

$$
1+\frac{a_{1} z}{1}+\frac{a_{2} / z}{1}+\frac{a_{8} z}{1}+\frac{a_{4} / z}{1}+\cdots
$$

where $z=r e^{i \theta}$ is a complex variable. This continued fraction reduces to (1.1) when $z=1$. We shall suppose that the coefficients $a_{n}$ satisfy the hypotheses of Theorem 3.1. It is clear then that for $|z| \leqq 1+\delta_{1}$, where $\delta_{1}=\epsilon /(k-\epsilon)$,

$$
\left|a_{2 n-1} z\right| \leqq k .
$$

Further, since the numbers $a_{2 n}$ lie outside the limaçon $r=2\left(k+\epsilon_{1}\right.$ $-\cos \theta$ ), positive numbers $\eta$ and $\delta_{2}$ can be chosen so small that for $|\theta| \leqq \eta$ and $r \leqq 1+\delta_{2}$ we have $a_{2 n} / z=R e^{i \phi}$ satisfying the conditions

$$
R_{2 n} \geqq 2\left(k-\cos \phi_{2 n}\right) \text {. }
$$

Let $\delta$ be the smaller of $\delta_{1}$ and $\delta_{2}$. In the open region $D$ defined by the inequalities

$$
D: \quad|\theta|<\eta, \quad 0<|z|<1+\delta
$$

the approximants of the continued fraction (3.5) are a family of rational functions of $z$. By (3.6) and (3.7) Lemma 3.1 asserts that the 
values of these approximants lie in the bounded region $V_{1}$. Thus this family is a normal family for $z$ in $D$.

We shall now show that this normal family converges in an open subset of $D$. Denote by $h$ the smaller of the two numbers $1 / 4(k-\epsilon)$ and $8\left(k+\epsilon_{1}-1\right) / 25$. Then for $|z| \leqq h$, the elements of (3.5) have the property that

$$
\left|a_{2 n-1} z\right| \leqq 1 / 4, \quad\left|a_{2 n} / z\right| \geqq 25 / 4 \quad(n=1,2, \cdots) .
$$

Thus by the theorem referred to concerning inequalities (1.2), the continued fraction (3.5) converges for $|z| \leqq h$ and hence in the intersection of this circular region with the region $D$. By Montel's [1] generalization of the Stieltjes-Vitali theorem the sequence of approximants of (3.5) converges (uniformly) in every closed region interior to $D$. Thus, in particular, this family converges for $z=1$.

The proof of the theorem is complete.

The following corollary is an immediate consequence of the theorem. The notation is that of the theorem.

Corollary 3.1. If

$$
r_{2 n} \geqq 2\left(k+\epsilon_{1}+1\right), \quad r_{2 n-1} \leqq k-\epsilon \quad(n=1,2, \cdots),
$$

the continued fraction (1.1) converges.

It is a consequence of Theorem 3.1 that if the hypotheses of this theorem hold, the continued fraction (2.1) converges at least in the wider sense, and converges to a finite value if and only if the continued fraction (1.1) converges to a value not equal to 1 .

The following result is thus established. The notation is that of the theorem.

COROLlary 3.2. If the elements $a_{n}=r_{n} e^{i \theta n}$ are such that

$$
r_{2 n-1} \geqq 2\left(k+\epsilon_{1}-\cos \theta_{2 n-1}\right), \quad r_{2 n} \leqq k-\epsilon \quad(n=1,2, \cdots),
$$

the continued fraction (1.1) converges at least in the wider sense.

We proceed with the proof of the following result.

Corollary 3.3. If the elements $a_{2 n}$ lie in the circle $|z| \leqq 1$ and if the quantities $a_{2 n+1}$ are outside of and bounded away from the cardioid $r=2(1-\cos \theta)$ the continued fraction (1.1) converges.

To prove this one observes that one can choose numbers $k>1$ and $\epsilon_{1}>0$ such that the numbers $a_{2 n+1}$ lie outside the limaçon $r=2\left(k+\epsilon_{1}-\cos \theta\right)$. A positive number $\epsilon$ can be chosen so that $\left|a_{2 n}\right| \leqq k-\epsilon$. The conditions of Theorem 3.1 are then fulfilled. 


\section{BIBLIOGRAPHY}

1. P. A. Montel, Legons sur les familles normales de fonctions analytique et leurs applications, Paris, 1927.

2. Walter Leighton and H. S. Wall, On the transformation and convergence of continued fractions, Amer. J. Math. vol. 58 (1936) pp. 267-281.

3. Walter Leighton, Convergence theorems for continued fractions, Duke Math. J. vol. 5 (1939) pp. 298-308.

4. W. T. Scott and H. S. Wall, Value regions for continued fractions, Bull. Amer. Math. Soc. vol. 47 (1941) pp. 580-585.

5. Walter Leighton and W. J. Thron, Continued fractions with complex elements, Duke Math. J. vol. 9 (1942) pp. 763-772.

6. W. J. Thron, Two families of twin convergence regions for continued fractions, Duke Math. J. vol. 10 (1943) pp. 677-685.

The Rice Institute 